\title{
QUEEN'S
UNIVERSITY
BELFAST
}

\section{Approaching the Intrinsic Limit in Transition Metal Diselenides via Point Defect Control}

Edelberg, D., Rhodes, D., Kerelsky, A., Kim, B., Wang, J., Zangiabadi, A., Kim, C., Abhinandan, A., Ardelean, J., Scullion, D., Embon, L., Zu, R., Santos, E. J. G., Balicas, L., Marianetti, C., Barmak, K., Zhu, X., Hone, J., \& Pasupathy, A. N. (2019). Approaching the Intrinsic Limit in Transition Metal Diselenides via Point Defect Control. Nano Letters. https://doi.org/10.1021/acs.nanolett.9b00985

\section{Published in:}

Nano Letters

\section{Document Version:}

Peer reviewed version

Queen's University Belfast - Research Portal:

Link to publication record in Queen's University Belfast Research Portal

\section{Publisher rights}

Copyright @ 2019 American Chemical Society.

This work is made available online in accordance with the publisher's policies. Please refer to any applicable terms of use of the publisher

\section{General rights}

Copyright for the publications made accessible via the Queen's University Belfast Research Portal is retained by the author(s) and / or other copyright owners and it is a condition of accessing these publications that users recognise and abide by the legal requirements associated with these rights.

Take down policy

The Research Portal is Queen's institutional repository that provides access to Queen's research output. Every effort has been made to ensure that content in the Research Portal does not infringe any person's rights, or applicable UK laws. If you discover content in the Research Portal that you believe breaches copyright or violates any law, please contact openaccess@qub.ac.uk. 


\title{
Approaching the Intrinsic Limit in Transition Metal Diselenides via Point Defect Control
}

\author{
D. Edelberg ${ }^{1,+}$, D. Rhodes ${ }^{2,+}$, A. Kerelsky ${ }^{1}$, B. Kim², J. Wang ${ }^{3}$, A. Zangiabadi ${ }^{5}$, C. Kim ${ }^{5}$, A. \\ Abhinandan', J. Ardelean ${ }^{1}$, M. Scully ${ }^{4}$, D. Scullion ${ }^{4}$, L. Embon ${ }^{1}$, I. Zhang ${ }^{1}$, R. Zư ${ }^{5}$, Elton J. G. \\ Santos $^{4}$, L. Balicas ${ }^{5,6}$, Chris Marianetti ${ }^{7}$, K. Barmak ${ }^{2, \#}$, X.-Y. Zhu ${ }^{3, \%}$, J. Hone ${ }^{2,+}$, A. N. Pasupathy ${ }^{1, *}$ \\ ${ }^{1}$ Department of Physics, Columbia University, New York, NY 10027, USA \\ ${ }^{2}$ Department of Mechanical Engineering, Columbia University, New York, NY 10027, USA \\ ${ }^{3}$ Department of Chemistry, Columbia University, New York, NY 10027, USA \\ ${ }^{4}$ School of Mathematics and Physics, Queen's University Belfast, BT7 1NN, UK \\ ${ }^{5}$ National High Magnetic Field Laboratory, Florida State University, Tallahassee, Florida 32310, \\ USA. \\ ${ }^{6}$ Department of Physics, Florida State University, Tallahassee, Florida 32306, USA \\ ${ }^{7}$ Department of Applied Physics and Applied Math, Columbia University, New York, NY \\ 10027, USA
}

\begin{abstract}
Two dimensional (2D) transition-metal dichalcogenide (TMD) based semiconductors have generated intense recent interest due to their novel optical and electronic properties, and potential for applications. In this work, we characterize the atomic and electronic nature of intrinsic point defects found in single crystals of these materials synthesized by two different methods - chemical vapor transport and self-flux growth. Using a combination of scanning tunneling microscopy (STM) and scanning transmission electron microscopy (STEM), we show that the two major intrinsic defects in these materials are metal vacancies and chalcogen antisites. We show that by control of the synthetic conditions, we can reduce the defect concentration from above $10^{13} / \mathrm{cm}^{2}$ to below $10^{11} / \mathrm{cm}^{2}$. Because these point defects act as centers for non-radiative recombination of excitons, this improvement in material quality leads to a hundred-fold increase in the radiative recombination efficiency.
\end{abstract}

The semiconducting transition metal dichalcogenides (TMDs) are a family of layered compounds with the formula $\mathrm{MX}_{2}(\mathrm{M}=[\mathrm{Mo}, \mathrm{W}]$ and $\mathrm{X}=[\mathrm{S}, \mathrm{Se}, \mathrm{Te}])$, which can be isolated in monolayer form and have promise in a wide range of applications in electronics and optoelectronics ${ }^{1,2,3,4}$. These materials host novel phenomena such as valley physics $^{5,6}$, interlayer tunneling ${ }^{7,8}$, topological properties ${ }^{9,10}$, and exciton superfluidity ${ }^{5}$ which are of fundamental interest and may enable new device functionality. However, crystalline disorder obscures intrinsic phenomena and imposes an upper limit on achievable functionality ${ }^{5,6,11,12}$. In particular, point defects ${ }^{13,14,15,16}$ strongly impact TMD monolayers: these defects cause carrier scattering and localization ${ }^{17-19}$, act as centers for non-radiative recombination $^{20-23}$, and give rise to localized eWSe2mission from excitonic traps ${ }^{24,25}$. Pioneering transport ${ }^{7,26,27}$ and STEM studies ${ }^{13,14,15}$ have explored the atomic nature and electronic impact of defects arising in monolayer TMDs, and indicate that the quality of these materials remains far behind the classic semiconducting materials such as $\mathrm{Si}$ and GaAs. Addressing the quality of these materials is urgently needed to advance their science and engineering applications.

In two-dimensional materials, disorder can arise from both intrinsic sources, such as point defects and grain boundaries in the crystal itself; and extrinsic sources arising from the environment, such as inhomogeneous strain, and charge traps / adsorbates in the substrate ${ }^{14,28}$. In the case of mechanically exfoliated graphene, the intrinsic defect density is extremely low $\left(10^{9}-10^{10} / \mathrm{cm}^{29,30}\right)$, and reducing extrinsic disorder by encapsulation in hexagonal boron nitride $(\mathrm{hBN})$ has enabled spectacular advances in device performance ${ }^{31-33}$. However, other 2D materials do not necessarily possess graphene's ultrahigh purity. In the case of semiconducting TMDs, $\mathrm{hBN}$ encapsulation also results in improved performance ${ }^{34,35}$, but the physical properties of these devices are still far from their theoretical 
limits, indicating that intrinsic disorder plays an important role. This is consistent with studies showing point defect densities exceeding $10^{12} / \mathrm{cm}^{2}$ in commonly used TMD materials ${ }^{36}$. Therefore, continued progress in the field necessitates the characterization, quantification, and minimization of defects in TMD materials. Toward this end, a particular challenge is the diversity of material sources, which include natural or synthesized single crystals, and large-area films grown by chemical vapor deposition (CVD) $)^{37,38}$, metal organic chemical vapor deposition ${ }^{39}$, physical vapor deposition ${ }^{40}$, and molecular beam epitaxy ${ }^{41}$, each of which can give rise to a different density and type of defects. For instance, STEM imaging of $\mathrm{MoS}_{2}$ reveals that CVD-grown films are dominated by S-vacancies, whereas defects in natural $\mathrm{MoS}_{2}$ crystals are predominantly Mo vacancies ${ }^{14}$.

In this study, we focus on the quality of synthesized TMD single crystals. Currently, single crystals remain the source of the highest-quality TMD monolayers ${ }^{11,37,42,43}$, and do not suffer from grain boundaries and phase separation $^{16,44,45}$ observed in large-area films. While much initial work on TMDs has utilized naturally occurring minerals ${ }^{7,46}$, laboratory-synthesized crystals provide a wider materials selection, and can offer a higher degree of quality control and reproducibility. Toward this end, a number of companies are currently supplying synthesized TMD crystals for laboratory use. However, synthesized TMD crystals have not been well characterized, and virtually no experimental work has examined the correlation between defect density and optoelectronic properties of monolayers derived from these bulk crystals. In this work, we use STM and STEM imaging to determine the type and density of intrinsic defects present in single crystals of $\mathrm{MoSe}_{2}$ and $\mathrm{WSe}_{2}$ synthesized by the chemical vapor transport (CVT) and flux growth techniques. Transition metal vacancies and anti-sites are found to be the mostcommon defect types, with Se vacancies being much rarer. Flux growth achieves 1-2 orders of magnitude lower defect density than CVT does, and defect densities lower than $10^{11} / \mathrm{cm}^{2}$ from our flux-grown sample are by far the lowest reported for TMD materials. This improvement is reflected in reduction of band-edge disorder measured by scanning tunneling spectroscopy, and orders of magnitude increase in photoluminescence quantum yield.

The CVT technique utilizes a transport agent, usually a halogen, to transport starting materials from a hot region into a cooler growth region where they form crystals ${ }^{47}$. CVT provides large crystals in a relatively short growth time at moderate temperatures, and thus has become the prevalent technique for TMD synthesis. The self-flux method in which crystals are grown directly from the molten phase is an alternative method known to create higherquality, albeit smaller, crystals ${ }^{48}$. In this work, we characterize three types of crystals: commercially obtained crystals grown by CVT and tested without further annealing (as-grown CVT or ag-CVT); crystals synthesized in our furnaces by CVT then annealed in a temperature gradient (treated CVT or t-CVT); and crystals grown by the self-flux method (flux). Details of the growth procedures, temperatures, and cooling rates are given in the methods section.

We first examine defects in bulk crystals through scanning tunneling microscopy (STM), which can provide defect lattice positions, local electronic structure, and defect density. To avoid surface contamination, crystals were cleaved in situ under UHV conditions. Examining a $25 \mathrm{~nm}$ square region of $\mathrm{MoSe}_{2}$ (Figure 1a), we observe two defect types that can be initially identified by contrast as either "dark" or "bright". As discussed further below, these two predominant types of defects account for the vast ( $>99 \%$ ) majority of defects imaged in both flux and CVT samples. Figures $1 \mathrm{~b}$ and $1 \mathrm{c}$ show atomic resolution images of these defects. The bright defects, which we denote $\mathrm{X}$, are located on a selenium site (Fig. 1b). Since there is no missing atom associated with this defect, it is not a selenium vacancy ${ }^{36,49}$, but is rather a substitutional impurity on the chalcogen site. Se vacancies can indeed be observed by STM but are roughly two orders of magnitude less common than the vacancies and substitutional impurities (see Supplementary Information section S1 for images of chalcogen vacancies). Further insight into this defect can be obtained by looking at the electronic structure using scanning tunneling spectroscopy (STS). Figure $1 \mathrm{~d}$ shows a sequence of tunneling spectra measured at varying distances from a single - $\mathrm{X}$ defect, out to a distance of $3.5 \mathrm{~nm}$. Directly over the defect, we measure a broad resonance pinned to the edge of the conduction band. The resonance shifts as a function of distance from the defect site, likely due to band bending effects ${ }^{50}$. This indicates that the $-X$ defects behave as n-type dopants. STM itself cannot identify the chemical nature of defects. However, we have observed that the concentration of $-X$ defects can vary widely between crystals depending on the growth method (see below), even when the same starting raw materials are used in the syntheses. This indicates that the 
defect is not associated with a foreign substituent, but is most likely an antisite defect, i.e, a Mo atom substituting for a Se atom as suggested by theoretical calculations (see below). The antisites are observed in roughly equal numbers on the top and bottom selenium layers of the $\mathrm{MX}_{2}$ unit cell as expected (see supplementary information section S6 for more details).

The dark defects observed in STM images, which we denote $-\mathrm{M}$, are aligned with the Mo sites, which are located in the center of a triangle of selenium atoms (Fig. 1c). In contrast to the $-\mathrm{X}$ defects, the $-\mathrm{M}$ defects show a resonance near the edge of the valence band, as shown in Fig. 1e. This indicates that the $-\mathrm{M}$ defects are electron acceptors. Defects spectra are further compared to spectra taken at $10 \mathrm{~nm}$ away as shown in supplemental information S2. Interestingly, whereas flux-grown $\mathrm{MoSe}_{2}$ possesses both $-\mathrm{M}$ and $-\mathrm{X}$ defects, similarly grown $\mathrm{WSe}_{2}$ displays only $-\mathrm{M}$ defects (see supplementary information S3 for atomic-resolution images of defects in $\mathrm{WSe}_{2}$ ). We note that the apparent heights of the defects that we see in STM images is strongly bias dependent, consistent with previous STM measurements on monolayer TMDs on graphite substrates ${ }^{51}$. This allows direct comparison of defects seen in STM and STEM. In order to perform STEM measurements, we exfoliate flakes of monolayer TMDs which are transferred onto holey carbon substrates as described in the Methods section. Shown in Fig. If is an STEM image that shows the only type of point defect observed in the flux-grown $\mathrm{WSe}_{2}$ sample (see supplementary information S4 for addition STEM images). The bright atoms in this image are $\mathrm{W}$ due to its high atomic number, indicating that the defect is a missing $\mathrm{W}$ atom, i.e., a metal vacancy. This chemical assignment of the $-\mathrm{M}$ defect is consistent with the STS observation that they act as electron acceptors.

We quantify the defect density by large-area STM imaging. Figures 2a-c show topographic scans $(0.5 \mu \mathrm{m} \times 0.5$ $\mu \mathrm{m}$ ) of the three $\mathrm{MoSe}_{2}$ materials under study. In the ag-CVT sample (Fig. 2a), the defect density is high enough such that the individual point defects have overlapping electronic signatures, and therefore STM can only provide a lower bound on the defect density of $>10^{13}-\mathrm{cm}^{-2}$ (1\% of unit cells). This defect density is dramatically reduced, to $(2.5 \pm 1.5) \times 10^{12}-\mathrm{cm}^{-2}(0.2 \%)$ in the t-CVT sample (Fig. 2b). The self-flux crystals display still lower defect density of $(1.7 \pm 0.5) \times 10^{11} \mathrm{~cm}^{-2}(0.01 \%)$ (Fig. 2c). See supplementary information S5 for defect counting procedures and counts. As for $\mathrm{MoSe}_{2}$, commercial ag-CVT WSe 2 exhibits a very high defect density and STM imaging can only provide a lower bound of $>10^{12} \mathrm{~cm}^{-2}(0.1 \%)$ (Fig. 2d). In the flux-grown $\mathrm{WSe}_{2}$, the defect density is dramatically smaller, $(7.0 \pm 2.2) \times 10^{10} \mathrm{~cm}^{-2}(0.006 \%)$ (Fig. 2e). This defect density is by far the lowest reported for any TMD semiconductor. We additionally sort defect counts by type for each growth method, a summary of these results for each growth are compared in Table 1. Additional sorting of defects is followed in the supplemental information S6 regarding the placement of each chalcogen defect within a single TMD layer. We note that in our imaging measurements, clear point defect impurities are only observed from the top $\mathrm{MX}_{2}$ layer (as determined by the apparent heights of the defects discussed above). Extended corrugations over larger scales are seen in large scale topographic images such as those shown in figure 2, which could arise from defects buried between layers ${ }^{52}$ or in deeper layers.

Our STM and STS measurements can be compared to theoretical expectations of defect formation energies and electronic structure from density functional theory (DFT). Here we found that a metal vacancy requires an additional $5.22 \mathrm{eV}$ per defect site for its formation (see Methods section for definitions of formation energies and chemical potentials). Using the Kröger-Vink (K-V) formalism to examine the chemistry of this defect gives a charge state of $4^{-}$when referenced to the neutral crystal causing this defect type to act as an electron acceptor (i.e., $\square_{\mathrm{M}}{ }^{4-}+4 h^{\bullet}$, where $\square_{\mathrm{M}}^{4-}$ denotes the vacancy on the metal site for a compound with chemical formula $\mathrm{MX}_{2}$ - this defect has a negative charge relative to the filled metal site in the neutral crystal reference state typically used in the K-V formalism, and $h^{\bullet}$ denotes the requisite holes in the valence band to maintain overall charge neutrality). DFT predictions for the local density of states match nicely with the observed STS, finding a shallow acceptor state (not pictured). The metal antisite defect has a formation energy of $4.81 \mathrm{eV}$. These defects exhibit a charge state of $6^{+}$ when referenced to the neutral crystal making them electron donors, consistent with STM and STS (i.e., $M_{X}^{6+}+6 e^{\prime}$ where $M_{X}^{6+}$ denotes the metal antisite and $e^{\prime}$ represents the electrons required to retain charge neutrality). Apart from the two observed defect types in experiments, we also calculate the formation energy for chalcogen vacancies. 
We find this energy to be $1.81 \mathrm{eV}$, which is lower than that of a metal vacancy or antisite. The observed lack of these vacancies in spite of their lower formation energy suggests that kinetics plays a large factor in determining observed defect concentrations. Details of these calculations can be found in the methods and a table of calculated values can be seen in the supplemental information. Additional experiments that control for the kinetics of the reaction (for example by species availability) are required to relate the observed defect concentrations to the formation energies discussed here.

From the observed defect densities and binding energies, we can use semiconductor theory to calculate the chemical potential in various samples of $\mathrm{MoSe}_{2}$ as a function of temperature ${ }^{53}$. The result of this calculation is shown in Figure $2 \mathrm{f}$ for the t-CVT and flux-grown samples. For the CVT material, we expect a relatively constant chemical potential due to large but compensated numbers of donor and acceptor defects. The flux-grown crystal, in contrast, has smaller defect density but a dominance of donors, resulting in a strong chemical potential shift towards the conduction band edge as a function of temperature. These considerations indicate that we should measure chemical potential shifts in our STS spectra between different samples. In order to measure this with high spatial resolution, we use STS spectroscopy to measure the local semiconducting gap at every pixel of a $256 \times 256$ px grid overlaid on a $0.5 \times 0.5 \mathrm{um}^{2}$ area. At each point, we extract a local value of the conduction and valence band edges from the local spectrum (see supplementary information S8 for a detailed description of the procedure). A sum of these two values gives the local value of the semiconducting bandgap.

Shown in Figure $3 \mathrm{a}$ is a color scale image of the measured bandgap variation in a t-CVT crystal. Color variations in this picture represent gap variations in the vicinity of defects. The average bandgap in this crystal is $860 \mathrm{meV}$, with defect-induced gap variations of order $50 \mathrm{meV}$. The bulk bandgap is in reasonable agreement with the theoretical gap of $840 \mathrm{meV}^{54}$. The variation seen for the t-CVT crystal is to be contrasted with a similar gap variation image for the flux crystal shown in figure $3 \mathrm{~b}$. While the flux crystal also exhibits an average bandgap of $860 \mathrm{meV}$, it displays much smaller variations in space due to the lower concentration of defects. To visualize these differences, histograms detailing the spread of gap sizes for t-CVT and flux are plotted in figure $3 \mathrm{c}$ and figure $3 \mathrm{~d}$ respectively. The observed gap variation in the t-CVT $(3 \sigma)$ is $50 \mathrm{meV}$, while the flux crystal shows a gap variation of $20 \mathrm{meV}$. As part of our measurement, STS was able to extract the valence and conduction band edges separately. Therefore, we further the analysis by examining the impact defects have on the valence and conduction band edges. In figure $3 \mathrm{e}, 3 \mathrm{f}$ we plot the valence band onset distributions for t-CVT (centered at $-370 \mathrm{meV}$ ) and flux (centered at $-760 \mathrm{meV}$ ) respectively. From these plots we see that most of the gap variation arises from defect states on the valence edge, which we found earlier correspond to metal vacancies. A similar analysis of the conduction band edge is plotted for $\mathrm{t}-\mathrm{CVT}$ in figure $3 \mathrm{~g}$ (centered at $490 \mathrm{meV}$ ) and flux in figure $3 \mathrm{~h}$ (centered at $100 \mathrm{meV}$ ). Here we see almost no variation, especially in the case of the flux crystal.

Our detailed gap maps can also be used to estimate the position of the chemical potential relative to the gap midpoint from $\mu=-\frac{\left\langle E_{C}\right\rangle+\left\langle E_{V}\right\rangle}{2}$. For the t-CVT sample, this gives a chemical potential $60 \mathrm{meV}$ below the gap center, indicating slight p-type doping. The self-flux crystals have a chemical potential of $330 \mathrm{meV}$ above the gap center, making them n-type. At $77 \mathrm{~K}$, we expect from figure $2 \mathrm{f}$ that the chemical potential should be $50 \mathrm{meV}$ below the gap center for t-CVT and $320 \mathrm{meV}$ above the gap center for self-flux. This agreement with STS mapping over a $500 \times 500 \mathrm{~nm}^{2}$ region indicates that we have properly accounted for all of the dopants in the semiconductor. Additionally, the observed behavior explains the commonly observed p-type ambipolar FET devices ${ }^{55}$ that have been made from t-CVT crystals. To visualize this further average $\mathrm{dI} / \mathrm{dV}$ spectra for each bandgap map are presented on the same plot in supplemental information S9.

To connect the large disparity of crystal imperfections versus growth observed across different crystals in STM to the monolayer limit, we carry out photoluminescence (PL) measurements as a simple way to measure the radiative response versus its quality. We isolate single layers through mechanical exfoliation from bulk single crystals ${ }^{37,42,43}$. Monolayers were simultaneously exfoliated from ag-CVT, t-CVT and flux grown crystals and each sample was subsequently handled under identically conditions to eliminate extrinsic factors. Each monolayer was encapsulated in $\mathrm{BN}$ and placed on a passivated $\mathrm{SiO}_{2}$ surface ${ }^{34}$. The resulting stacks were measured under the same conditions 
(laser excitation power, spot size, and acquisition time). Additional data was taken to ensure that these curves were taken within the linear regime of the laser, as is seen in supplemental information S10. The raw PL data for $\mathrm{MoSe}_{2}$ at $4 \mathrm{~K}$ are plotted on a $\log$ scale in figure $4 \mathrm{a}$. While the peak position shifts very slightly as crystalline quality is enhanced, the linewidth (FWHM) shows an obvious decrease with improved crystal quality, from 4 meV in agCVT to $3 \mathrm{meV}$ in t-CVT to $2 \mathrm{meV}$ in self-flux sample. The decrease in FWHM is consistent with the improvement in homogeneity of monolayer sample as defect density decreases. A dramatic effect is seen in the total light emission intensity (proportional to the quantum yield), with the self-flux sample having a 10-fold increase in light intensity over the t-CVT monolayer, and a 100-fold increase over the ag-CVT monolayer. We note that improvements in PL yield can also be achieved by various surface treatments ${ }^{56,57}$ that can affect the exciton lifetime or light absorption. In our PL experiments, we take care to keep the environment of the films as identical as possible across samples via encapsulation in BN. Our measurements are also performed in the linear regime as shown in the supplementary information. The large suppression of excitons with little change to peak position suggests that defects provide nonradiative pathways for the recombination of excitons, via exciton localization ${ }^{58}$ or defect enhanced Auger processes $^{20,22,23}$, lowering the PL yield from the intrinsic limit of a pristine sample.

To quantify the impact of defects on the excitonic properties, we extend the PL measurements described above to various temperatures on t-CVT and flux monolayers (ag-CVT is omitted due to a lack of intensity at high temperatures). We plot the data taken for both $\mathrm{MoSe}_{2}$ (red) and $\mathrm{WSe}_{2}$ (blue) on a log scale at $77 \mathrm{~K}$ in figure $4 \mathrm{~b}$ where $\mathrm{t}-\mathrm{CVT}$ is dashed and flux is solid. Since the major observed difference from the crystals is the overall PL intensity, we plot the integrated PL intensity as seen for $\mathrm{MoSe}_{2}$ in figure 4c. To model the shape of the integrated PL signal we must account for the unique band structure of monolayer TMDs. In the TMD materials, both the valence and conduction bands are spin split due to spin-orbit coupling. The magnitude of the splitting in the valence band is roughly an order of magnitude larger than the conduction-band splitting $(\sim 300 \mathrm{meV}$ versus $\sim 30 \mathrm{meV})$. Due to the spin splitting in the conduction band, one of the two transitions from the conduction band to the upper valence band (A exciton) is dark, while the other is optically bright. In $\mathrm{MoSe}_{2}$ the lower of the two transitions is bright, while the situation is reversed in $\mathrm{WSe}_{2}$. We fit the integrated PL intensity to an empirical Arrhenius equation, which accounts for both the spin split exciton ${ }^{59}$, and non-radiative processes which average multiple recombination rates. The full analysis followed is described in the supplemental information S11. We find that the integrated PL emission can be described as follows for $\mathrm{MoSe}_{2}$ :

$$
I_{T o t}=\frac{1}{1+C_{1} * e^{-\frac{\Delta E_{N R}}{k_{B} T}}+C_{2} * e^{-\frac{\Delta E_{D a r k}}{k_{B} T}}}
$$

Here the non-radiative term arises both from defects and phonon scattering at higher temperatures, and other term arises from thermal equilibrium between the dark and bright excitons. In figure $4 \mathrm{c}$ a sudden drop can be seen in the PL signal of the self-flux $\mathrm{MoSe}_{2}$ monolayer above roughly $60 \mathrm{~K}$. This can be attributed to the Boltzmann distribution of electrons able to access the dark exciton state. As per our fit prescribed above we extract the dark exciton energy to be $40 \mathrm{meV}$ above the bright exciton binding energy. This matches well with reports utilizing a backgate to directly measure the dark exciton at $30 \mathrm{meV}$ above the bright one ${ }^{60}$.

Like $\mathrm{MoSe}_{2}$, the PL resonances of $\mathrm{WSe}_{2}$ have roughly the same peak position across growth methods. The temperature dependent integrated intensity shows distinct behaviors for the flux versus CVT crystals as shown in figure $4 \mathrm{~d}$. The self-flux crystal shows an initial increase in the PL intensity with decreasing temperature down to about $150 \mathrm{~K}$, below which the intensity drops sharply. The CVT crystal however shows a decreasing intensity with decreasing temperature starting from room temperature. This difference in the case of $\mathrm{WSe}_{2}$ arises due to the fact that the lowest energy transition is dark ${ }^{61}$. This implies that at sufficiently low temperature only the dark state is populated, exponentially suppressing the PL intensity. At high enough temperature, on the other hand, the PL intensity is suppressed with increasing temperature due to phonon scattering. We can model both effects together using the same formula as $\mathrm{MoSe}_{2}$ with a sign change: 


$$
I_{T o t}=\frac{1}{1+C_{1} * e^{-\frac{\Delta E_{N R}}{k_{B} T}}+C_{2} * e^{\frac{\Delta E_{D a r k}}{k_{B} T}}}
$$

This change of sign indicates that the dark exciton is now at a lower energy than the bright. Thus, PL shows a maximum at some intermediate temperature which is determined by the interplay between defect and phonon recombination versus dark exciton coupling. Using the fitting for the integrated intensity from the flux crystal, we extract a conduction-band splitting of $43 \mathrm{meV}$. Our measurement of this splitting is within experimental error of the value directly measured from magnetic field measurements $(47 \mathrm{meV})^{62,63}$. We additionally find that CVT crystals exhibit stronger defect mediated coupling to the dark exciton state diminishing the PL signal out to much higher temperatures.

Our studies of defects in transition-metal dichalcogenides show definitively that there is a direct link between intrinsic point defect concentration in bulk crystals and the optoelectronic properties of exfoliated monolayers. The improvements in synthesis presented here have led to a lowering of the defect concentration by two orders of magnitude when compared to the current state of the art. Such improvements are a necessary step towards achieving many of the predicted optical phenomena that require high exciton concentration as well as transport phenomena that require long scattering times. We note in conclusion that while our synthetic achievements set a new benchmark for TMD semiconductors, the lowest bulk defect concentrations achieved here $\left(\sim 10^{18} / \mathrm{cm}^{3}\right)$ are still significantly higher than those achieved in the best III-V semiconductor films, indicating that there is still room for refining synthetic processes to achieve higher quality TMD materials. 


\section{Methods}

\section{Flux growth}

$\mathrm{MoSe}_{2}$ and $\mathrm{WSe}_{2}$ crystals were synthesized by reacting Mo/W powders, 99.999\%, with Se shot, 99.999\%, typically in a ratio of 1:20. These materials were first loaded into a quartz ampoule. A piece of quartz wool is then pressed into a cylindrical shape and pushed into the quartz ampoule, approximately $1 \mathrm{~cm}$ above the raw elements. The ampoule was then evacuated and sealed at $\sim 10^{-3}$ Torr. For growth, the ampoule is heated to $1000{ }^{\circ} \mathrm{C}$ over 48 hours, held there for 3 days, then cooled at a rate of $1.5^{\circ} \mathrm{C}$ down to $400{ }^{\circ} \mathrm{C}$ and subsequently flipped and centrifuged. Crystals are then harvested from the quartz wool filter and annealed at a temperature of $250{ }^{\circ} \mathrm{C}$ with the empty end of the quartz ampoule held approximately at $100^{\circ} \mathrm{C}$ for 48 hours.

\section{CVT}

$\mathrm{MoSe}_{2}$ crystals were synthesized by reacting Mo powder, $99.999 \%$, with Se shot, $99.999 \%$, in stoichiometric proportions with iodine $99.999 \%$ as a transport agent. These materials were first loaded into a quartz ampoule 12 $\mathrm{cm}$ in length, $1 \mathrm{~cm}$ in diameter, then evacuated and sealed at $\sim 10^{-3}$ Torr. For growth, the ampoule is heated to $1000{ }^{\circ} \mathrm{C}$ over a period of 48 hours, held there for 1 week, then cooled for 3 days to $750{ }^{\circ} \mathrm{C}$ and subsequently quenched in air. Crystals are then harvested and rinsed in acetone and isopropanol to remove iodine residue, and left to dry.

\section{Ab initio density functional theory methods}

First-principle calculations for defect formation energies were done using density functional theory (DFT) within the projected augmented wave method ${ }^{64,65}$, as implemented in the VASP code ${ }^{66,67}$. The generalized gradient approximation ${ }^{68}$ is employed to treat exchange and correlation in DFT. Projected augmented wave method (PAW) was used in the description of the bonding environment for $\mathrm{W}, \mathrm{Mo}$, and Se. The structures are fully relaxed until all interatomic forces are smaller than $0.02 \mathrm{eV} / \AA$. The Brillouin zone was sampled with a $5 \times 5 \times 1 \mathrm{k}$-mesh under the Monkhorst-Pack scheme ${ }^{69}$. Plane-wave energy cut offs of $400 \mathrm{eV}$ and $500 \mathrm{eV}$ are used for structural relaxation and static runs, respectively. The defect formation energies are defined via $E_{\text {form }}=E_{\text {defect }}\left(E_{\text {pristine }}+\sum n_{i} \mu{ }_{i}\right)$, where $E_{\text {defect }}$ stands for the total energy of a defected monolayer, $E_{\text {pristine }}$ is the total energy of a pristine monolayer, $n_{i}$ is the number of removed (minus sign) or added (plus sign) species $i$ and $\mu_{\mathrm{i}}$ is the chemical potential of species $i$. The size of the supercell was determined by convergence tests, resulting in a $6 \times 6 \times 1$ with a $15 \AA$ vacuum space. The chemical potentials of each species are constrained by the relation ${ }^{69}, \mu_{\mathrm{MoSe} 2}=\mu_{\mathrm{Mo}}+2 \mu \mathrm{se}$, where $\mu_{\mathrm{Mo}}$ and $\mu_{\mathrm{Se}}$ are the chemical potentials for Mo and Se, respectively; $\mu$ MoSe2 is the total energy per formula unit of $\mathrm{MoSe}_{2}$. We determine the range of chemical potentials with two extreme cases: Mo-rich environment and Se-rich environment. For the Mo-rich environment, $\mu$ Mo is chosen to be the total energy per atom of Mo in the bcc structure. For the Serich environment, $\mu \mathrm{se}$ is chosen to be the total energy per atom of Se in the trigonal phase. The defect formation energies as a function of $\mu_{\text {Mo }}$ are presented in the supplementary information S7. The values in the main text are extracted with the chemical potentials following $\mu_{\mathrm{i}}=\mathrm{E}_{\mathrm{i}}+\mathrm{E}_{\text {bond, }}$, where $\mathrm{E}_{\mathrm{i}}=$ Total energy of bulk metal or crystal of chalcogenide and $\mathrm{E}_{\mathrm{bond}}=\left(\mathrm{E}_{\mathrm{MX} 2}-\mathrm{E}_{\mathrm{M}}-2 \mathrm{E}_{\mathrm{X}}\right) / 3^{70}$.

\section{Scanning Tunneling Microscopy and Spectroscopy (STM)}

STM measurements were performed using a custom built, variable temperature, UHV STM system. Single crystals of $\mathrm{MoSe}_{2}$ and $\mathrm{WSe}_{2}$ were mounted onto metallic sample holders using a vacuum safe silver paste. Samples were then transferred into the STM chamber and cleaved in-situ, exposing a clean surface. A Pt-Ir STM tip was cleaned and calibrated against a gold (111) single crystal prior to the measurements. Measurements were collected at $82 \mathrm{~K}$ and $300 \mathrm{~K}$.

\section{Optical measurements}


Optical stacks of $\mathrm{BN} / \mathrm{TMD} / \mathrm{BN}$ were fabricated using the polypropylene carbonate (PPC) method as described in

${ }^{71}$ and placed on passivated $\mathrm{SiO}_{2}{ }^{34}$. MoSe 2 samples were measured using a closed-cycle He cryostat (Attocube Attodry 1100) and an excitation wavelength of $532 \mathrm{~nm}$ using a cw diode laser with an approximate power of 2.0 $\mu \mathrm{W}$. For $\mathrm{WSe}_{2}$, samples were loaded into a cryostat with a sapphire window which is combined with a homemade photoluminescence setup with an excitation wavelength of $532 \mathrm{~nm}$ using a cw diode laser and a power of $80 \mu \mathrm{W}$. For cooling, either helium-4 or liquid nitrogen were continuously flowed through the cryostat chamber, immersing the sample while temperature was modulated with a stage heater.

\section{Transmission Electron Microscope (TEM) sample preparation and Scanning Transmission Electron Microscope (STEM) Imaging}

For the preparation of electron transparent samples for point defect density measurements, the TMD crystals were mechanically exfoliated using Scotch ${ }^{\mathrm{TM}}$ tape. The exfoliated flakes were transferred onto oxidized silicon wafer substrates. The monolayer flakes of the exfoliated TMD crystals were identified using light optical microscopy. The monolayers were then transferred onto Quantifoil ${ }^{\circledR}$ holey carbon TEM grids using isopropyl alcohol as a medium. After the alcohol evaporated, the holey carbon grid was attached to the wafer and the monolayer. The wafer sections were then slowly immersed in $1 \mathrm{M}$ potassium hydroxide $(\mathrm{KOH})$ solution to etch the very top surface of the oxide and release the TEM grid and the exfoliated crystals attached to it. Distilled water was used to dilute and wash away the $\mathrm{KOH}$ solution from the TEM grids. As a last step, TEM grids were immersed in warm $\left(40{ }^{\circ} \mathrm{C}\right)$ acetone for 10 minutes to dissolve any residue that remained from the exfoliation and sample transfer.

The STEM imaging of TMD monolayers was carried out in an FEI Talos F200X instrument operated at $200 \mathrm{kV}$. A low beam current $(\sim 60 \mathrm{pA})$ was used to reduce the amount of beam damage. By using the smallest condenser aperture $(50 \mu \mathrm{m})$ and beam size 9 , the convergence angle of the probe was calculated to be approximately $10 \mathrm{mrad}$. Images were acquired at a series of times to determine the rate at which metal vacancies formed in the samples when irradiated by the electron beam. For $\mathrm{MoSe}_{2}$, the density of metal vacancies was found to increase linearly with time allowing extrapolation to time zero to determine the initial density. For $\mathrm{WSe}_{2}$, no new metal vacancies were found to be created up to 40 seconds of imaging. Thus, for these samples, images from multiple regions were collected using a 20 second acquisition time. To improve the contrast and reduce the noise in the images for quantification of point defect density, the Butterworth filter in Gatan Digital Micrograph and the Wiener deconvolution in MATLAB were used. In addition, the simulated structure of the monolayer using the CrystalMaker software was overlaid on the processed STEM images to aid in identification of the metal and the chalcogen positions.

\section{Acknowledgements}

Yinan Dong, Bryan Medina and Steven French are acknowledged for their assistance in sample preparation. This work is supported by the NSF MRSEC program through Columbia in the Center for Precision Assembly of Superstratic and Superatomic Solids (DMR-1420634). Support for STM instrumentation is provided by the Air Force Office of Scientific Research (grant number FA9550-16-1-0601). KB and AZ acknowledge partial funding support from the Dean of the School of Engineering and Applied Science at Columbia University. DS acknowledges his EPSRC studentship. EJGS acknowledges the use of computational resources from the UK national highperformance computing service (ARCHER) for which access was obtained via the UKCP consortium (EPSRC grant ref EP/K013564/1); the UK Materials and Molecular Modeling Hub for access to THOMAS, which is partially funded by EPSRC (EP/P020194/1). EJGS also acknowledges the Queen's Fellow Award through the grant number M8407MPH, the Enabling Fund (A5047TSL), and the Department for the Economy (USI 097). L.B acknowledges the US Army Research Office MURI grant W911NF-11-1-0362 (Synthesis and Physical Characterization of TwoDimensional Materials and Their Heterostructures) and the Office Naval Research DURIP Grant 11997003 (Stacking Under Inert Conditions). Preliminary growth and characterization single crystals were performed at the 
National High Magnetic Field Laboratory, which is supported by the NSF Cooperative Agreement DMR-1157490 and the State of Florida. 

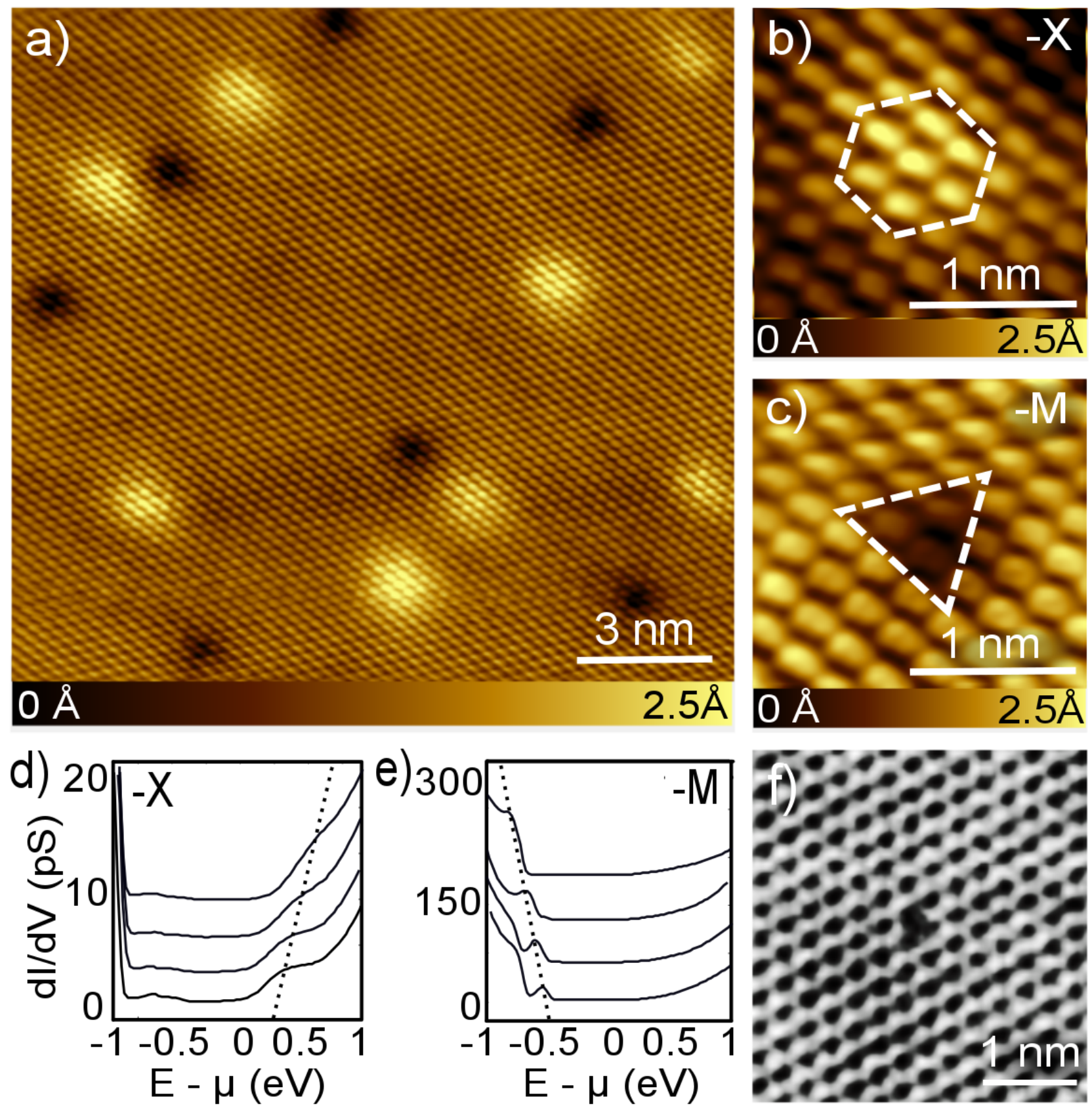

Figure 1 : Defect atomic and electronic structure

High resolution STM topographic images of $\mathrm{MoSe}_{2}(\mathrm{~V}=1.25 \mathrm{~V} \mathrm{I}=100 \mathrm{pA})$ showing a) a $25 \mathrm{~nm}$ area with two types of defects $b$ ) a single chalcogen antisite, $-X$ type defect and c) a single metal vacancy, $-M$ type defect. Differential conductance curves obtained at various distances from a d) chalcogen antisite, showing the presence of a donor state on the defect site, and e) single metal vacancy, showing the presence of an acceptor state on the defect site f) STEM imaging confirming that $-\mathrm{M}$ defects are metal vacancies 

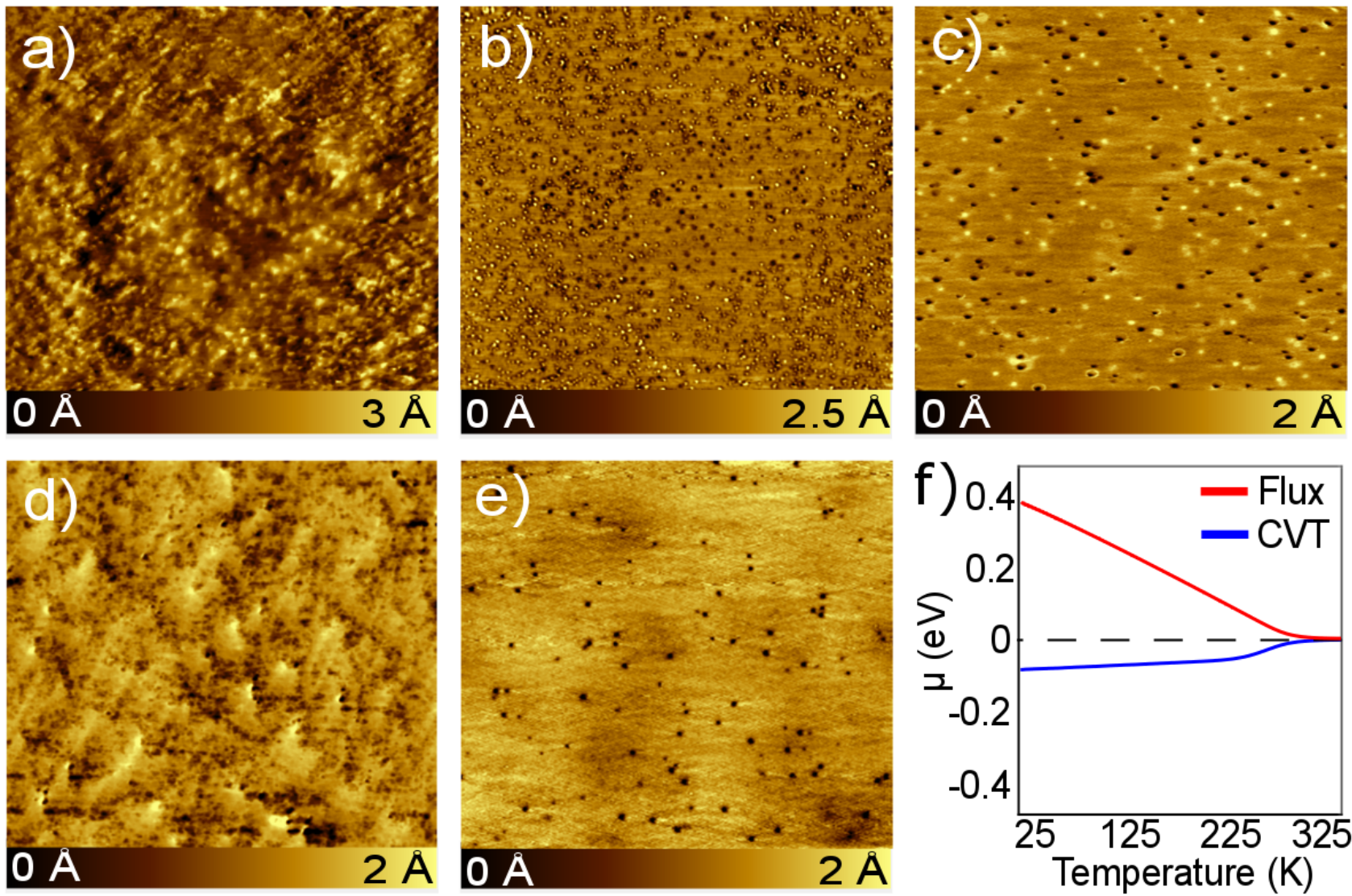

$0 \AA$

$2.5 \AA 0 \AA$

$2 \AA$
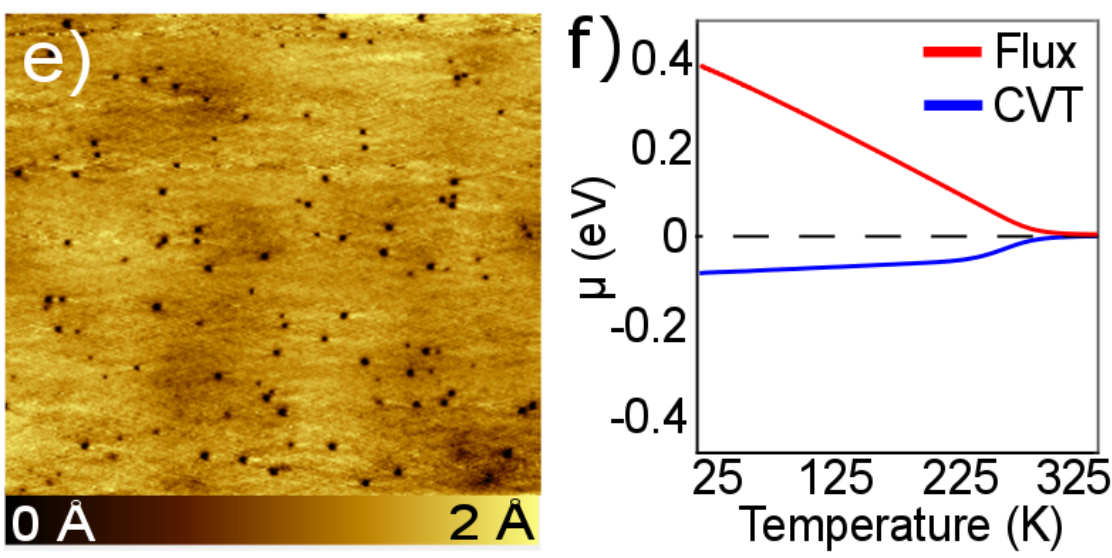

Figure 2 : Defect density versus growth method in $\mathrm{MoSe}_{2}$ and $\mathrm{WSe}_{2}$

STM topographic images of $500 \times 500 \mathrm{~nm}^{2}$ areas of an $\mathrm{MoSe}_{2}$ crystal grown by a) commercial as-grown CVT b) post-annealed t-CVT, and c) self-flux. The imaging conditions for a-c) were a STM bias of $1.25 \mathrm{~V}$ and current of 100pA. STM topographic images of $500 \times 500 \mathrm{~nm}^{2}$ areas of WSe $\mathrm{W}_{2}$ crystals grown by d) commercial CVT (V=1V I=100 pA) and e) self-flux ( $V=0.75 \mathrm{~V} \mathrm{I=150} \mathrm{pA).} \mathrm{f)} \mathrm{Chemical} \mathrm{potential} \mathrm{calculated} \mathrm{from} \mathrm{defect}$ densities for $\mathrm{MoSe}_{2}$ on treated t-CVT and self flux crystals as a function of temperature. 

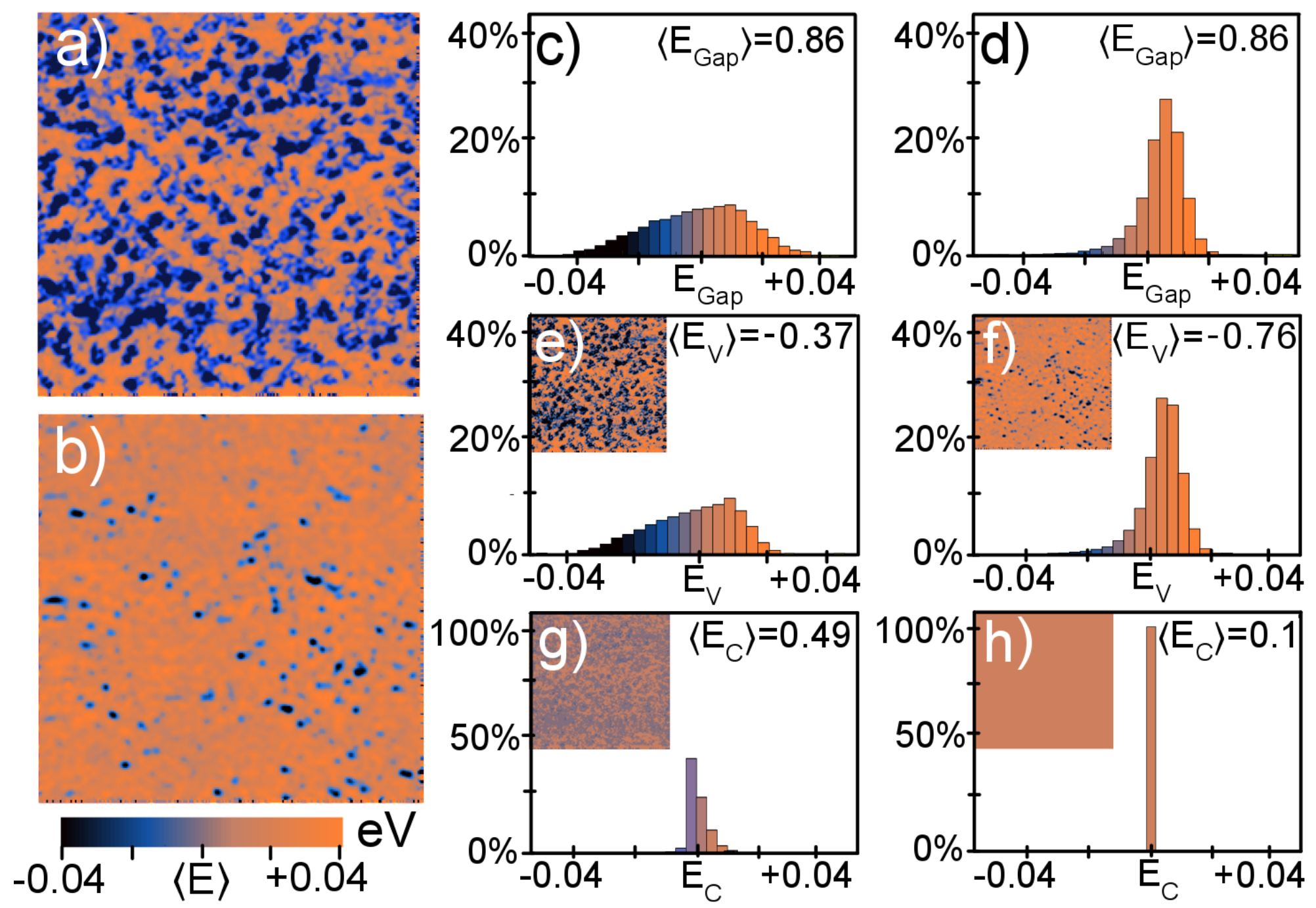

Figure 3: Spatially Resolved Bandgap by Scanning Tunneling Spectroscopy

Map of the semiconducting gap measured on a $256 \times 256$ pixel grid over a $500 \times 500 \mathrm{~nm}^{2}$ region plotted in the same color scale of a) post annealed t-CVT and b) self-flux grown $\mathrm{MoSe}_{2}$ crystal at $82 \mathrm{~K}$. c-d) Histograms of bandgap sizes based on $a$ ) and $b$ ) respectively. Histograms of e-f) valence band edge and g-h) conduction band edge from t-CVT and self-flux respectively. The insets to e-h) are measured images of the respective band edges in the same color scale. 
a)

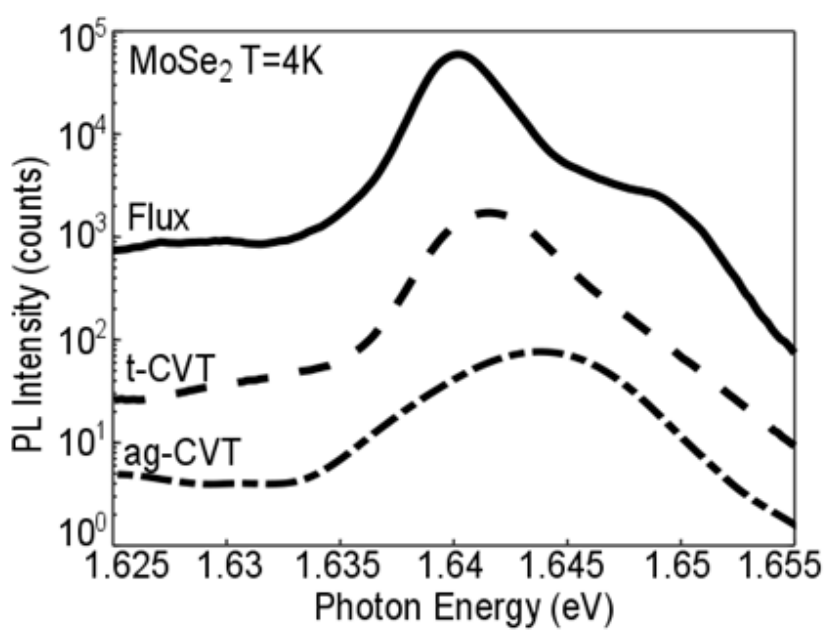

c)

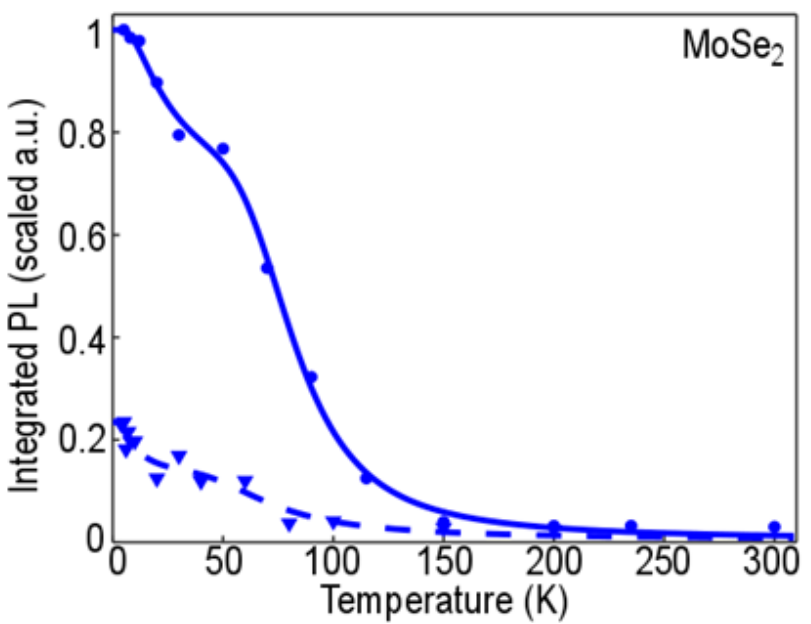

b)

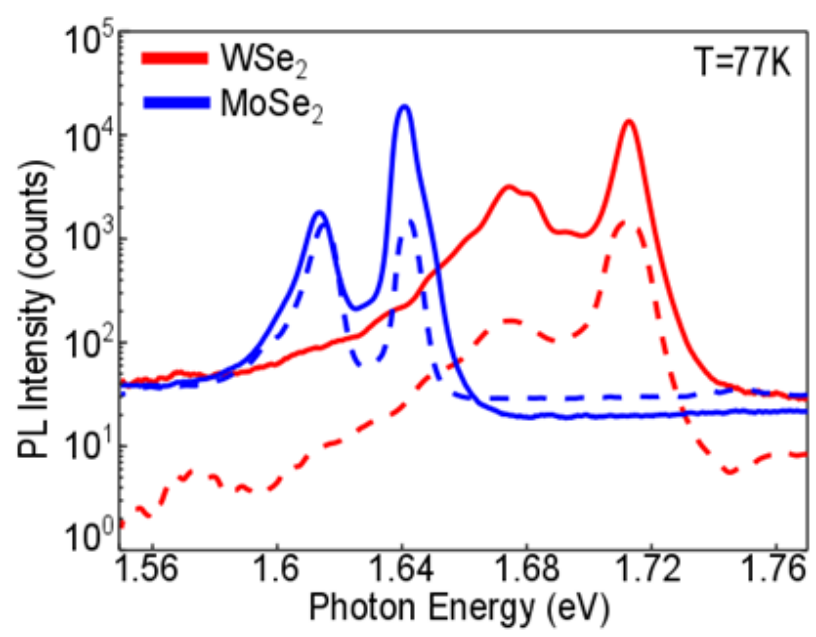

d)

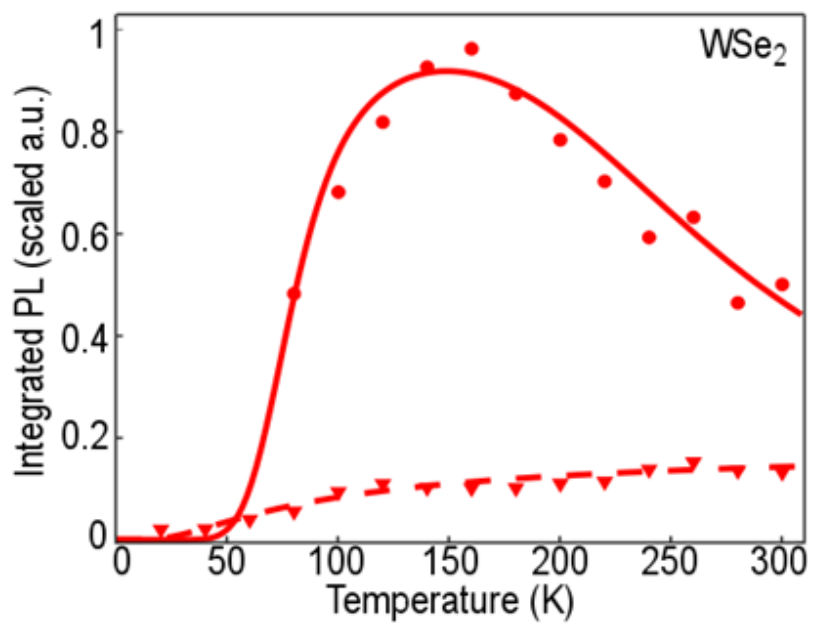

Figure 4: Temperature Dependent Photoluminescence of monolayer TMDs for different Growth Methods a) PL for ag-CVT, t-CVT and self-flux grown monolayers of $\mathrm{MoSe}_{2}$. All monolayers are encapsulated in $\mathrm{h}-\mathrm{BN}$ and measured under identical conditions at 4K. The intensity of the PL from t-CVT and ag-CVT have been multiplied by 6.7 and 275 respectively in order to match the peak intensity of the flux-grown monolayer b) PL comparing $\mathrm{t}-\mathrm{CVT}$ (dashed) and self-flux (solid) crystals of $\mathrm{MoSe}_{2}$ (red) and WSe $\mathrm{W}_{2}$ (blue) c) Temperature dependence of the integrated A-exciton PL intensity in $\mathrm{MoSe}_{2}$ for $\mathrm{t}$-CVT and flux-grown samples.d) Temperature dependence of the integrated A-exciton PL intensity in $\mathrm{WSe}_{2}$ for t-CVT and flux-grown samples. The drop in intensity at low temperature is due to the lowest excitonic transition being optically dark. The blue and red lines in b) and c) are fits to a modified Arrhenius form from which the conduction-band splitting can be extracted. 


\begin{tabular}{lccc}
\hline $\begin{array}{c}\text { Crystal Growth } \\
\text { Method }\end{array}$ & $\begin{array}{c}\text { Total Defect Count } \\
\text { Defects } / \mathrm{cm}^{2}\end{array}$ & $\begin{array}{c}\text { M-Site Defects } \\
\text { Defects } / \mathrm{cm}^{2}\end{array}$ & $\begin{array}{c}\text { X-Site Defects } \\
\text { Defects } / \mathrm{cm}^{2}\end{array}$ \\
\hline MoSe $_{2}$ ag-CVT & $>10^{13}$ & $>10^{13}$ & $>10^{13}$ \\
MoSe $_{2}$ t-CVT & $(2.5 \pm 1.5) \times 10^{12}$ & $(1.3 \pm 0.8) \times 10^{12}$ & $(1.12 \pm 0.6) \times 10^{12}$ \\
MoSe $_{2}$ Self-flux & $(1.7 \pm 0.5) \times 10^{11}$ & $(7.0 \pm 0.2) \times 10^{10}$ & $(9.91 \pm 0.2) \times 10^{10}$ \\
\hline WSe $_{2}$ ag-CVT & $>10^{12}$ & $>10^{12}$ & $>10^{11}$ \\
WSe $_{2}$ Self-flux & $(7.0 \pm 2.2) \times 10^{10}$ & $(6.41 \pm 2.0) \times 10^{10}$ & $(5.83 \pm 1.8) \times 10^{9}$ \\
\hline
\end{tabular}

Table 1: For each crystal studied, total defect density and distribution of defect types. The error bars are from sample to sample variation across different batches of crystals grown by the same growth method. Within a single growth batch, the statistical variations across the crystals are much smaller than the sample-to-sample variations across growth batches. 


\section{References}

1 Xu, X., Yao, W., Xiao, D. \& Heinz, T. F. Spin and pseudospins in layered transition metal dichalcogenides. Nat Phys 10, 343-350, doi:10.1038/nphys2942 (2014).

2 Chen, X., Yan, T., Zhu, B., Yang, S. \& Cui, X. Optical Control of Spin Polarization in Monolayer Transition Metal Dichalcogenides. ACS nano (2017).

3 Lee, J., Mak, K. F. \& Shan, J. Electrical control of the valley Hall effect in bilayer MoS2 transistors. Nat Nano 11, 421-425, doi:10.1038/nnano.2015.337

http://www.nature.com/nnano/journal/v11/n5/abs/nnano.2015.337.html - supplementary-information (2016).

$4 \quad$ Mak, K. F. \& Shan, J. Photonics and optoelectronics of 2D semiconductor transition metal dichalcogenides. Nat Photon 10, 216-226, doi:10.1038/nphoton.2015.282 (2016).

5 Fogler, M., Butov, L. \& Novoselov, K. High-temperature superfluidity with indirect excitons in van der Waals heterostructures. arXiv preprint arXiv:1404.1418 (2014).

6 Sie, E. J., Frenzel, A. J., Lee, Y.-H., Kong, J. \& Gedik, N. Intervalley biexcitons and many-body effects in monolayer \$\{\mathrm\{MoS\}\}_\{2\}\$. Physical Review B 92, 125417 (2015).

7 RadisavljevicB, RadenovicA, BrivioJ, GiacomettiV \& KisA. Single-layer MoS2 transistors. Nat Nano 6, 147150, doi:http://www.nature.com/nnano/journal/v6/n3/abs/nnano.2010.279.html - supplementaryinformation (2011).

$8 \quad$ Ma, N. \& Jena, D. Interband tunneling in two-dimensional crystal semiconductors. Applied Physics Letters 102, 132102 (2013).

9 Soluyanov, A. A. et al. Type-Il weyl semimetals. Nature 527, 495-498 (2015).

10 Bruno, F. Y. et al. Observation of large topologically trivial Fermi arcs in the candidate type-II Weyl semimetal WT e 2. Physical Review B 94, 121112 (2016).

11 Choi, W. et al. Recent development of two-dimensional transition metal dichalcogenides and their applications. Materials Today (2017).

12 Zeng, Q. \& Liu, Z. Novel Optoelectronic Devices: Transition-Metal-Dichalcogenide-Based 2D Heterostructures. Advanced Electronic Materials (2018).

13 Lin, Z. et al. Defect engineering of two-dimensional transition metal dichalcogenides. 2D Materials 3, 022002 (2016).

14 Hong, J. et al. Exploring atomic defects in molybdenum disulphide monolayers. Nat Commun 6, doi:10.1038/ncomms7293 (2015).

15 Zhou, W. et al. Intrinsic structural defects in monolayer molybdenum disulfide. Nano letters 13, 26152622 (2013).

16 Yankowitz, M., McKenzie, D. \& LeRoy, B. J. Local Spectroscopic Characterization of Spin and Layer Polarization in \$\{\mathrm\{WSe\}\}\{2\}\$. Physical Review Letters 115, 136803 (2015).

17 Walukiewicz, W. Carrier scattering by native defects in heavily doped semiconductors. Physical Review $B$ 41, 10218 (1990).

18 El-Mahalawy, S. \& Evans, B. Temperature dependence of the electrical conductivity and hall coefficient in 2H-MoS2, MoSe2, WSe2, and MoTe2. physica status solidi (b) 79, 713-722 (1977).

19 Fivaz, R. \& Mooser, E. Mobility of charge carriers in semiconducting layer structures. Physical Review 163, 743 (1967).

20 Wang, H., Zhang, C. \& Rana, F. Ultrafast dynamics of defect-assisted electron-hole recombination in monolayer MoS2. Nano letters 15, 339-345 (2014).

21 Palummo, M., Bernardi, M. \& Grossman, J. C. Exciton radiative lifetimes in two-dimensional transition metal dichalcogenides. Nano letters 15, 2794-2800 (2015).

22 Moody, G., Schaibley, J. \& Xu, X. Exciton dynamics in monolayer transition metal dichalcogenides. JOSA B 33, C39-C49 (2016). 
23 Wang, H. et al. Fast exciton annihilation by capture of electrons or holes by defects via Auger scattering in monolayer metal dichalcogenides. Physical Review B 91, 165411 (2015).

24 Bastard, G., Delalande, C., Meynadier, M., Frijlink, P. \& Voos, M. Low-temperature exciton trapping on interface defects in semiconductor quantum wells. Physical Review B 29, 7042 (1984).

$25 \mathrm{Ky}$, N. H. \& Reinhart, F. Amphoteric native defect reactions in Si-doped GaAs. Journal of applied physics 83, 718-724 (1998).

26 Ovchinnikov, D., Allain, A., Huang, Y.-S., Dumcenco, D. \& Kis, A. Electrical transport properties of singlelayer WS2. ACS nano 8, 8174-8181 (2014).

27 Baugher, B. W. H., Churchill, H. O. H., Yang, Y. \& Jarillo-Herrero, P. Intrinsic Electronic Transport Properties of High-Quality Monolayer and Bilayer MoS2. Nano Letters 13, 4212-4216, doi:10.1021/nl401916s (2013).

28 Raja, A. et al. Coulomb engineering of the bandgap and excitons in two-dimensional materials. Nature Communications 8, 15251 (2017).

29 Liu, L., Qing, M., Wang, Y. \& Chen, S. Defects in graphene: generation, healing, and their effects on the properties of graphene: a review. Journal of Materials Science \& Technology 31, 599-606 (2015).

30 Zhong, J.-H. et al. Quantitative correlation between defect density and heterogeneous electron transfer rate of single layer graphene. Journal of the American Chemical Society 136, 16609-16617 (2014).

31 Dean, C. R. et al. Boron nitride substrates for high-quality graphene electronics. Nature nanotechnology 5, 722-726 (2010).

32 Mayorov, A. S. et al. Micrometer-scale ballistic transport in encapsulated graphene at room temperature. Nano letters 11, 2396-2399 (2011).

33 Britnell, L. et al. Electron tunneling through ultrathin boron nitride crystalline barriers. Nano letters 12, 1707-1710 (2012).

34 Ajayi, O. et al. Approaching the Intrinsic Photoluminescence Linewidth in Transition Metal Dichalcogenide Monolayers. 2D Materials (2017).

35 Wang, J. I.-J. et al. Electronic transport of encapsulated graphene and WSe2 devices fabricated by pickup of prepatterned hBN. Nano letters 15, 1898-1903 (2015).

36 McDonnell, S., Addou, R., Buie, C., Wallace, R. M. \& Hinkle, C. L. Defect-dominated doping and contact resistance in MoS2. ACS nano 8, 2880-2888 (2014).

37 Lee, Y. H. et al. Synthesis of Large-Area MoS2 Atomic Layers with Chemical Vapor Deposition. Advanced Materials 24, 2320-2325 (2012).

38 Chen, J. et al. Chemical Vapor Deposition of Large-size Monolayer MoSe2 Crystals on Molten Glass. Journal of the American Chemical Society (2017).

39 Eichfeld, S. M. et al. Highly scalable, atomically thin WSe2 grown via metal-organic chemical vapor deposition. ACS nano 9, 2080-2087 (2015).

40 Muratore, C. et al. Continuous ultra-thin MoS2 films grown by low-temperature physical vapor deposition. Applied Physics Letters 104, 261604 (2014).

41 Zhang, Y. et al. Direct observation of the transition from indirect to direct bandgap in atomically thin epitaxial MoSe2. Nature nanotechnology 9, 111-115 (2014).

42 Van Der Zande, A. M. et al. Grains and grain boundaries in highly crystalline monolayer molybdenum disulphide. Nature materials 12, 554-561 (2013).

43 Roy, A. et al. Structural and electrical properties of MoTe2 and MoSe2 grown by molecular beam epitaxy. ACS applied materials \& interfaces 8, 7396-7402 (2016).

44 Kerelsky, A. et al. Absence of a Band Gap at the Interface of a Metal and Highly Doped Monolayer MoS2. Nano Letters (2017).

45 Kang, K. et al. High-mobility three-atom-thick semiconducting films with wafer-scale homogeneity. Nature 520, 656 (2015). 
Manzeli, S., Ovchinnikov, D., Pasquier, D., Yazyev, O. V. \& Kis, A. 2D transition metal dichalcogenides. 2, 17033, doi:10.1038/natrevmats.2017.33 (2017).

47 Ubaldini, A., Jacimovic, J., Ubrig, N. \& Giannini, E. Chloride-Driven Chemical Vapor Transport Method for Crystal Growth of Transition Metal Dichalcogenides. Crystal Growth \& Design 13, 4453-4459, doi:10.1021/cg400953e (2013).

48 Zhang, X. et al. Flux method growth of bulk MoS2 single crystals and their application as a saturable absorber. CrystEngComm 17, 4026-4032, doi:10.1039/C5CE00484E (2015).

49 Voiry, D., Mohite, A. \& Chhowalla, M. Phase engineering of transition metal dichalcogenides. Chemical Society Reviews 44, 2702-2712 (2015).

50 Stroscio, J. A., Feenstra, R. M. \& Fein, A. P. Local state density and long-range screening of adsorbed oxygen atoms on the GaAs(110) surface. Physical Review Letters 58, 1668-1671, doi:10.1103/PhysRevLett.58.1668 (1987).

51 Zhang, S. et al. Defect Structure of Localized Excitons in a WSe 2 Monolayer. Physical review letters 119, 046101 (2017).

52 Lu, C.-P., Li, G., Mao, J., Wang, L.-M. \& Andrei, E. Y. Bandgap, Mid-Gap States, and Gating Effects in MoS2. Nano Letters 14, 4628-4633, doi:10.1021/nl501659n (2014).

53 Jin, Z., Li, X., Mullen, J. T. \& Kim, K. W. Intrinsic transport properties of electrons and holes in monolayer transition-metal dichalcogenides. Physical Review B 90, 045422 (2014).

54 Tongay, S. et al. Thermally Driven Crossover from Indirect toward Direct Bandgap in 2D Semiconductors: MoSe2 versus MoS2. Nano Letters 12, 5576-5580, doi:10.1021/nl302584w (2012).

55 Pradhan, N. R. et al. Ambipolar molybdenum diselenide field-effect transistors: field-effect and hall mobilities. ACS nano 8, 7923-7929 (2014).

56 Amani, M. et al. Near-unity photoluminescence quantum yield in MoS2. Science 350, 1065-1068 (2015).

57 Amani, M. et al. High luminescence efficiency in MoS2 grown by chemical vapor deposition. ACS nano 10, 6535-6541 (2016).

58 Godde, T. et al. Exciton and trion dynamics in atomically thin MoSe 2 and WSe 2: Effect of localization. Physical Review B 94, 165301 (2016).

59 Baranowski, M. et al. Dark excitons and the elusive valley polarization in transition metal dichalcogenides. 2D Materials 4, 025016 (2017).

60 Quereda, J., Ghiasi, T. S., van Zwol, F. A., van der Wal, C. H. \& van Wees, B. J. Observation of bright and dark exciton transitions in monolayer MoSe 2 by photocurrent spectroscopy. 2D Materials (2017).

61 Dery, H. \& Song, Y. Polarization analysis of excitons in monolayer and bilayer transition-metal dichalcogenides. Physical Review B 92, 125431 (2015).

62 Zhang, X.-X., You, Y., Zhao, S. Y. F. \& Heinz, T. F. Experimental evidence for dark excitons in monolayer WSe 2. Physical review letters 115, 257403 (2015).

63 Wang, G. et al. Magneto-optics in transition metal diselenide monolayers. 2D Materials 2, 034002 (2015).

64 Kresse, G. \& Hafner, J. Ab initio molecular dynamics for open-shell transition metals. Physical Review $B$ 48, 13115 (1993).

65 Kresse, G. \& Furthmüller, J. Efficient iterative schemes for ab initio total-energy calculations using a plane-wave basis set. Physical review B 54, 11169 (1996).

66 Perdew, J. P., Burke, K. \& Ernzerhof, M. Generalized gradient approximation made simple. Physical review letters 77, 3865 (1996).

67 Blöchl, P. E. Projector augmented-wave method. Physical review B 50, 17953 (1994).

68 Kresse, G. \& Joubert, D. From ultrasoft pseudopotentials to the projector augmented-wave method. Physical Review B 59, 1758 (1999).

69 Monkhorst, H. J. \& Pack, J. D. Special points for Brillouin-zone integrations. Physical review B 13, 5188 (1976). 
70 Haldar, S., Vovusha, H., Yadav, M. K., Eriksson, O. \& Sanyal, B. Systematic study of structural, electronic, and optical properties of atomic-scale defects in the two-dimensional transition metal dichalcogenides M X 2 (M= Mo, W; X= S, Se, Te). Physical Review B 92, 235408 (2015).

71 Wang, L. et al. One-dimensional electrical contact to a two-dimensional material. Science 342, 614-617 (2013).

† These authors contributed equally to this work

Correspondence to:

*apn2108@columbia.edu

+jh2228@columbia.edu

\%xyzhu@columbia.edu

\#kb2612@columbia.edu 